\title{
Transcriptome reveals senescing callus tissue of Aquilaria malaccensis, an endangered tropical tree, triggers similar response as wounding with respect to terpenoid biosynthesis
}

\begin{abstract}
Aquilaria malaccensis is an endangered tree species listed in the Appendix II of CITES. It is a main source of highly valuable resinous wood known as agarwood, which is rich in secondary metabolites. De novo assembly of sequences produced by transcriptome sequencing using next-generation sequencing technologies offers a rapid approach to obtain expressed gene sequences for non-model organisms such as Aquilaria. To investigate the genes and pathways that might control molecular mechanism of $\mathrm{A}$. malaccensis under controlled environment, we sequenced two transcriptome libraries constructed from mRNAs of healthy and senescing callus tissues using Illumina sequencing. We obtained 200,062,275 and 166,544,202 reads for healthy and senescing callus libraries, respectively. We compiled 231,594 transcripts and identified 107,593 transcripts by similarity analysis against the National Center for Biotechnology Information (NCBI) public database. A total of 96,743 transcripts were functionally annotated using the Gene Ontology (GO) and Kyoto Encyclopedia of Genes and Genomes (KEGG) analyses. We assigned 46,076 of the transcripts to a total of 144 KEGG pathways. We focused on genes identified as contributing to fragrant compound synthesis and defense response pathways, which are important pathways leading to agarwood compound formation. This study provides abundant transcriptomic data and valuable sequence resources for future genomic studies on $\mathrm{A}$. malaccensis. This is the first report of callus transcriptome from A. malaccensis.
\end{abstract}

Keyword: Agarwood; Callus; Next-generation sequencing; Secondary metabolites; Senescence; Sesquiterpenoid 